\title{
NEW TECHNOLOGIES IN VASCULAR REHABILITATION
}

Prosperi Loris ${ }^{1}$, Barassi Giovanni ${ }^{2}$, Supplizi Marco ${ }^{1}$, Giancola Alessandra ${ }^{3}$, Di Matteo Celeste ${ }^{1}$, Manca Mara ${ }^{1}$, Gallo Laura ${ }^{1}$, Di Iorio Angelo ${ }^{4}$

'Physiotherapy, Rehabilitation and Rehabilitation Centre (Ce.Fi.R.R.), Venue "G.d'Annunzio" University, ChietiPescara (IT);

${ }^{2} \mathrm{PhD}$, Coordinator of the Physiotherapy, Rehabilitation and Rehabilitation Centre (Ce.Fi.R.R.) Venue "G. d'Annunzio "University, Chieti-Pescara (Italy)

${ }^{3}$ Ce.Fi.R.R. Gemelli Molise, "Catholic University of Sacred Heart”- Rome-Campobasso, professionalizing teaching center;

${ }^{4}$ Associate Professor, Department of Aging Sciences, “G.d'Annunzio” University, Chieti-Pescara.

KEYWORDS: Lymphedema, Intermittent Pneumatic Compression Devices, Bandages, Physiotherapy,

\section{ABSTRACT}

Lymphedema of lower limbs represents an important health problem, characterized by the necessity to identify new methods of treatment.

The aim of this study was to evaluate the efficacy of the treatment with Lymphatic Kinesiology Taping (LKT) or Intermittent Pneumatic Compression therapy (IPC) on a homogeneous group of 40 women affected by primary lymphedema within stage 2 of lower limbs, comparing the two methods. Patients were equally and randomly divided into a group A subjected to application of LKT and group B subjected to IPC. All patients were evaluated through electric bioimpedentiometry (analysis of Total Body Water and Extracellular Water) and circumferential measurement of lower limbs through a measurement tape, both before and after the protocol (T0 and T1).

Both treatment groups highlighted an improvement of the lymphedema, with a marked reduction of volume of lower limbs and a better hydric balance of the body.

LKT and IPC can be considered as viable options for the treatment of primary lymphedema within stage 2 of lower limbs.

\section{INTRODUCTION}

Lymphedema, defined as a condition of abnormal accumulation of lymphatic fluids in the subcutaneous interstices, is nowadays not only an extremely widespread and disabling condition, but also an extremely underestimated and under-treated problem (Lee et al., 2018).

Is possible to identify multiple causes of lymphedema, including genetic factors, environment and lifestyle factors, and traumatic factors (Lee et al., 2018). Among these also the typical immobility of elderly subjects or people suffering from neurological pathologies limiting movement should not be underestimated as a cause of inefficiency of the lymphatic system and consequent formation of secondary lymphedema (Lee et al., 2018).

In the rehabilitation field, it is possible to identify multiple treatment methods aimed at reducing lymphedema. The most common ones, identified today as a gold standard for the treatment of this pathology, are compression therapy using compression bandage or graduated compression elastic stockings and manual lymphatic drainage (Bjork and Hettrick, 2019).

However, new approaches to the treatment of oedemas have emerged over time, in many cases superimposable or complementary in terms of results compared to those indicated above (Bjork and Hettrick, 2019). Intermittent Pneumatic Compression therapy (IPC) has proven to be a valid tool in the treatment of distal oedemas (Rockson, 2018; Feldman et al., 2012). It usually consists of an air compressor connected by tubes to pants or bands, inside which air chambers are inserted which inflate sequentially according to a pressure gradient that can be set directly by the instrument, in distal-proximal direction, generating an intermittent external compression on the limb to be treated (Feldman et al., 2012).

Compression, in the most advanced systems, can be set according to the amount of $\mathrm{mmHg}$ that you want to apply for each air chamber. There is no clear consensus regarding the pressure gradients to be apply through IPC for the treatment of different pathological conditions related to the presence of edema of the limbs, with an extremely variable range of pressures ranging from a few tens of $\mathrm{mmHg}$ up to over 120 mmHG (Feldman et al., 2012). Anyway, it is assumed that an acceptable pressure range for an average application of the treatment should range between 25 and $70 \mathrm{mmHg}$, increasingly adapted to the increase in the extent and fibrosity of the edema to be treated (Feldman et al., 2012). The application times are also variable and to be commensurate with the extent of the problem to be treated, with an average duration per session of between 30 and 50 minutes and a variable treatment frequency, until reaching the two daily sessions for periods of a few weeks in particularly problematic pathological conditions (Feldman et al., 2012).

Many research highlighted positive effect from IPC therapy in presence of libs edemas of traumatic (Airaksinen et al., 1991), oncologic (Gurdal et al., 2012), venous-lymphatic (Aldrich et al., 2017) and neurologic-immobilization (Tessari et al., 2018) origin.

Another alternative method of treatment of limbs edema, with a growing success in the physiotherapy field, is the application of Lymphatic Kinesiology Taping 
(LKT) (Kase and Stockheimer, 2006).

This instrument consists of a cotton tape, spreaded with a not-medicated anallergic acrylic glue and capable of longitudinal stretch (Kase, 1996). The tape is water-resistant, with a long-lasting application directly on the skin and a thickness comparable to that of human skin. Its action depends on the kind of cut, shape and tension given to tape (Kase, 1996). It is supposed that the lymphatic drainage action of the tape depends on a lifting effect on the skin, which can increase the subcutaneous space in which lymphatic vessels can expand to increase lymphatic outflow (Kafa et al., 2015), as well as increasing the lymphatic capillary uptake by stretching $\mathrm{h}$ anchor filaments attached to the interstitium (Kase and Stockheimer, 2006). In this sense the application is made with single tape divided into stripes confluent on a single anchorage placed on a big lymphatic station, with the small stripes creating a canvas of multiple overlapped fan of tape able to stimulate the outflow of the lymph from the periphery to the nearest lymphatic station (Kase and Stockheimer, 2006). This method highlighted good results in the management of different kind of edemas (Thomaz et al., 2018; Kafa et al., 2015), although there is not univocal consent on the real efficacy of this method in its various applications (Pivetta et al., 2017, Teixeira et al., 2016).

Basing on these considerations, aiming to identify the real therapeutic potential of these alternative techniques for the management of edema of limbs, we decided to perform a comparative study on the efficacy of IPC and LKT in the management of primary lymphedema within stage 2 of lower limbs.

\section{MATERIALS AND METHODS}

Within the Physiotherapy Center of Rehabilitation and Reeducation (Ce.Fi.R.R.) located at the structures of the University "Gabriele d'Annunzio" of ChietiPescara, it has been performed a study to verify the efficacy of IPC and LKT in the management of primary lymphedema within stage 2 of lower limbs. The study was conducted in accordance with the etic principles of the Declaration of Helsinki.

A total of 40 women, affected by primary lymphedema within stage 2 of lower limbs, with a mean age of $39,7 \pm 9,7$ years old, were selected and randomized into two groups of treatment. Group A $(n=20)$ underwent treatment with application of LKT on lower limbs. Group B $(n=20)$ underwent treatment with IPC of lower limbs.

Exclusion criteria were the presence of phlebitis and wounds on lower limbs, blood hypo and hypertension, cardiovascular or renal diseases, risk of thrombosis, vagotomy, asthma, infections and neurologic pathologies.

Patients has been evaluated at time T0 (before starting the protocol) and time T1 (after the end of the protocol) through electric bioimpedentiometry (BIA 101 Anniversary, Akern S.r.1.C, Montacchiello, Italy) and circumferential measurements of lower limbs.

Bioimpedentiometry is an instrument for the evaluation of body composition and hydric balance of the body, which uses a low intensity alternated current traveling through a tetrapolar system of electrodes placed on the right side of the body, two on the dorsal side of the hand and two on the dorsal side of the foot (Ward, 2006; Abu Khaled et al., 1988). The route of this current through the body is then read by the instrument to give values for body resistance and reactance, which are then elaborated by a software to quantify the body composition (Ward, 2006; Abu Khaled et al.; 1988). For this study, we choose to consider values for Total Body Water (TBW) and Extracellular Water (ECW).

Circumferential measurement of limbs is a simple anthropometric system to evaluate the volume variations of a limb. It is performed with a measuring tape placed on specific symmetrical points of limbs (te Slaa et al., 2011). For this study we choose to take as point 0 the center of the kneecap of both lower limbs, measuring at $+5,+10,+15,+20$ and +25 cm cranially and at $-5,-10,-15,-20$ and $-25 \mathrm{~cm}$ caudally.

Patients of group A were treated with the application of LKT (TemTex®, Towatek Korea Co. Ltd., Gyeonggi-Do, Korea). The bandage was realized as following:

- measurement and cut, for both lower limbs, of two stripes long from the groin to the kneecap and two stripes long from the popliteal fossa to the insertional area of the Achilles tendon.

- cutting of each stripe into 5 smaller stripes confluent in a single anchor rounded at the angles (to create a sort of fan);

- application, on both thighs placed in abduction and external rotation with bended knee, of the anchor of a stripe on the medial groin area, without giving tension to the tape, and consequent fan application of smaller stripes to cover the whole anterior region of the thigh, with a tension of each small stripe of about $0 \%$ to $10 \%$ of the maximum stretch of the tape; following application of the second stripe, with the same method, starting from the lateral groin area, overlapping the first taping to create a net of tape on the anterior face of the thigh;

- application, on both legs placed in extension of knee and ankle with the patient in prone position, of the anchor of a stripe on the medial popliteal area, without giving tension to the tape, and consequent fan application of smaller stripes to cover the whole posterior region of the thigh, with a tension of each small stripe of about $0 \%$ to $10 \%$ of the maximum stretch of the tape; following application of the second stripe, with the same method, starting from the lateral popliteal area, overlapping the first taping to create a net of tape on the posterior face of the leg.

This LKT application was performed 2 times a week for 4 weeks, maintaining the bandage applied for 3 to 4 consecutive days between each application.

Patients in group B has been treated with the application of computerized IPC with 8 air chambers (Galaxy V BAC Technology S.r.l. (C, Figline Valdarno, Italy). The therapy was applied as following:

- the patient was stripped of his pants and socks, to wear a single-use protection pants for pressure therapy for hygienic purposes;

- accommodation of the patient supine on the bed, wearing a half body vest for the application of IPC on legs and trunk;

- $\quad$ setting of the "wave" application program, which consists in the sequential inflation of the 8 air chambers, each one maintaining the compression 


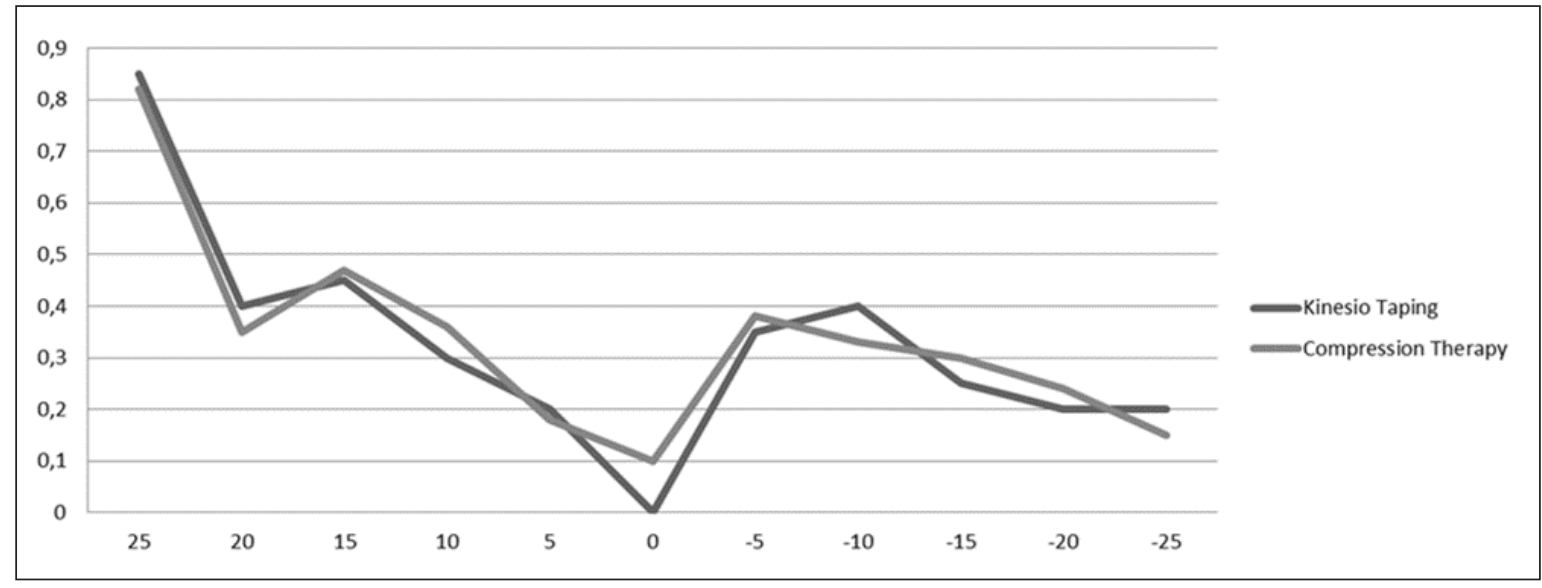

Fig. 1 - Mean circumference reduction of lower limbs in all detection points, regarding group A (LKT) and group B (IPC) (the $\mathrm{X}$ axis indicates the measurement point on lower limbs, expressed in $\mathrm{cm}$, and the $\mathrm{Y}$ axis indicates the circumference reduction value, expressed in $\mathrm{cm}$ ).

for the whole sequence, starting from the plantar fascia reaching the abdomen and then deflate all the air chambers together to start a new sequence;

- $\quad$ setting of the value of the 8 air chambers, simulating the decreasing pressure of a compression bandage or compression socks, starting from the foot reaching the abdomen with pressure values respectively of 70-65-60-55-50-45-40-35 $\mathrm{mmHg}$;

- $\quad$ execution of the therapy for a total time of 30 minutes for each session.

The application of IPC was done 3 times a week for 4 weeks, with 30 minutes sessions repeated every 2-3 days.

\section{RESULTS}

The quantitative analysis of collected data was performed using the Office Excel software (Microsoft ${ }^{\circledR}$, Redmond, Washington, USA), through which it was possible to realize the graphic representation of the mean variance of circumferences of lower limbs and TBW and ECW detected by the electric bioimpedentiometry.

Moreover, using the statistical analysis software

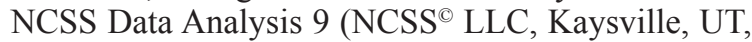
USA), it was possible to evaluate the significance of results obtained, using the Paired Sample T-Test.

The quantitative analysis of the variance of circumferences of lower limbs clearly highlights a reduction of volume of both lower limbs in both treated groups. It can be seen how the mean reduction of circumference in each point of lower limbs followed the same trend both in group A and in group B, with a reduction of volume more pronounced at the proximal level of the thighs and at the proximal level of the leg (Figure 1).

From a statistical standpoint variation of circumferences detected for lower limbs, even if quantitatively appreciable, resulted non-significant both in group A and in group B.

In quantitative terms is possible to highlight how the bioimpedentiometric analysis detected a reduction of TBW and ECW values both in group A and in group $B$, with a reduction value similar between groups (Figures 2.1 and 2.2).

From a statistical standpoint reductions obtained for TBW and ECW in group A and group B resulted significant, with a value of $\mathrm{p}<0,05$ (Figures 3.1, 3.2,4.1 and 4.2).

\section{DISCUSSION}

Obtained results proved the efficacy of LKT and IPC in the management of primary lymphedema within

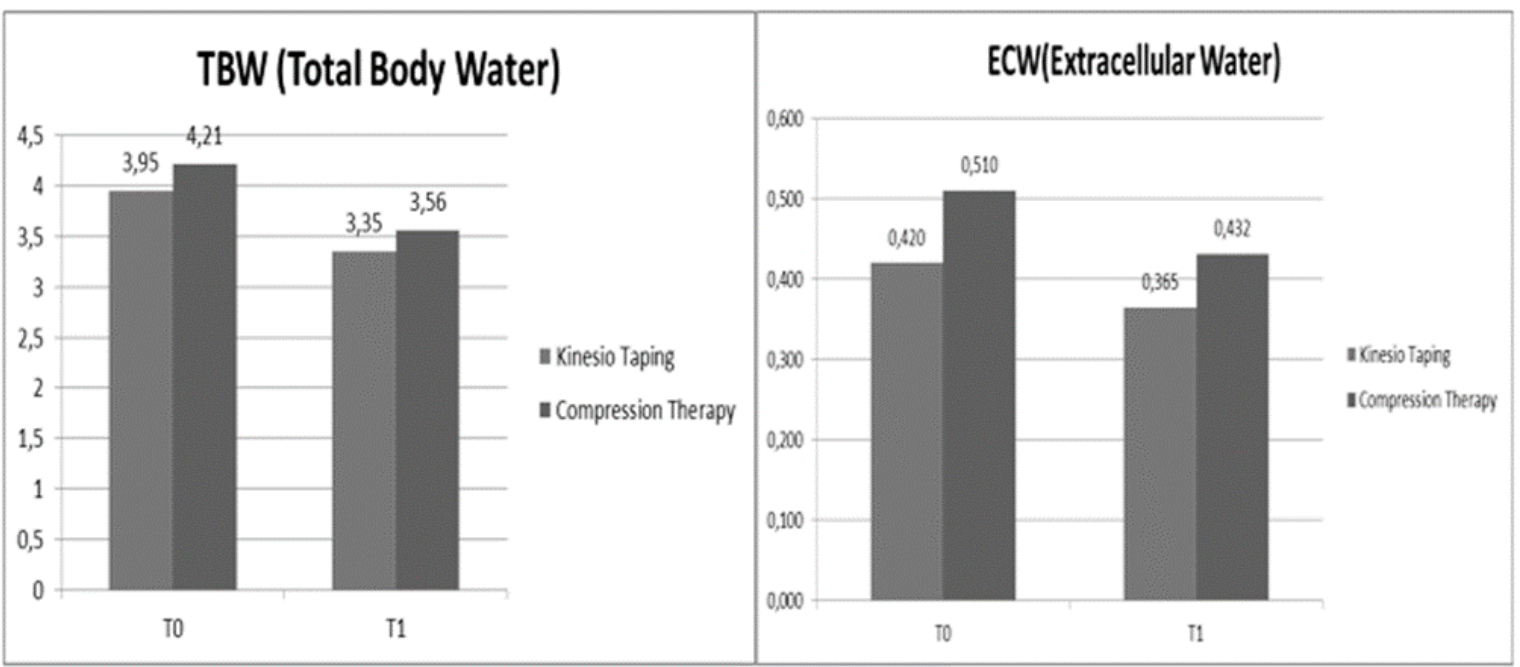

Fig. 2.1 , 2.2. - Mean reduction of total body water (TBW) and extracellular body water (ECW) for group A (LKT) and group B (IPC). 


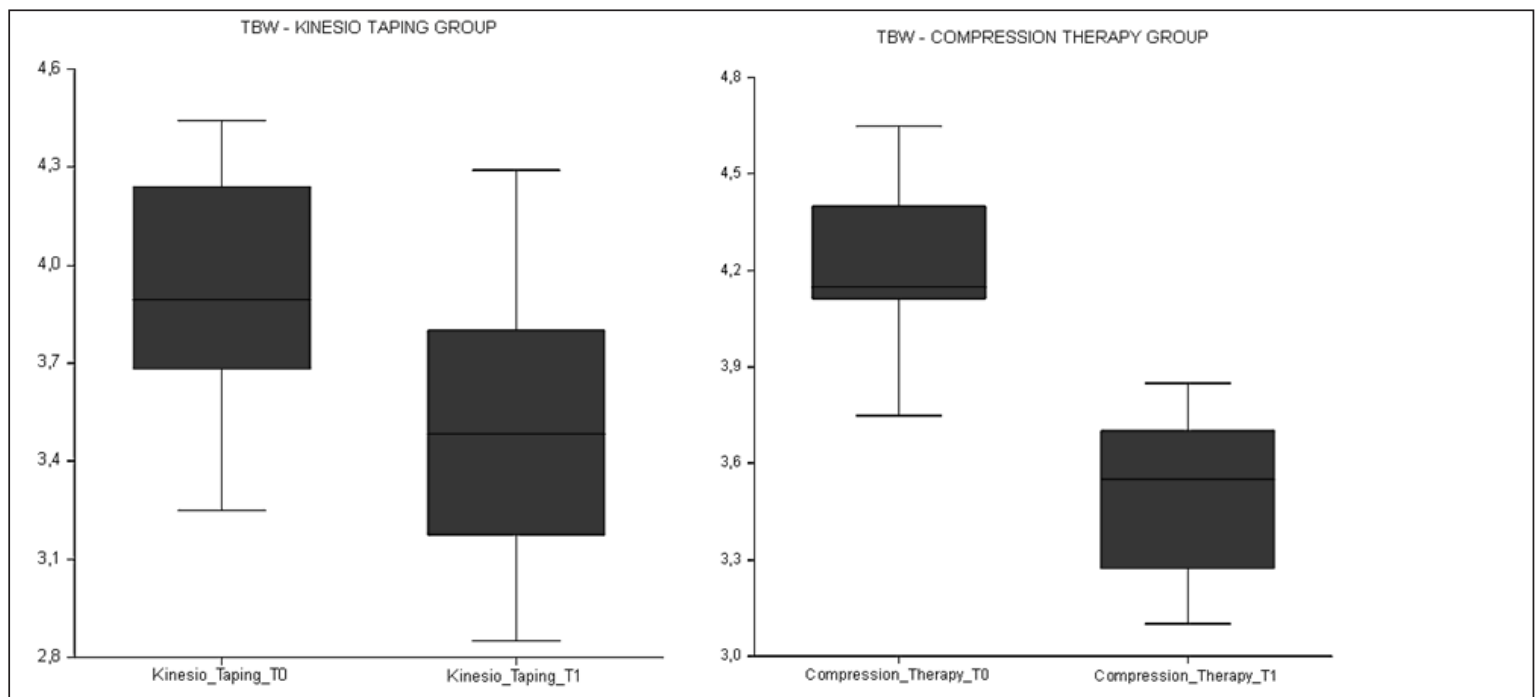

Fig. 3.1 , 3.2. - Statistical box plots of the mean variations of total body water (TBW) in group A (LKT) and group B (IPC).

stage 2 of lower limbs.

It seems that there is no difference in terms of therapeutic efficacy between the two systems, with these following the same reduction trend in terms of volume limbs and hydric balance.

Results obtained seems to confirm what is supported by the actual scientific literature, which often highlighted the efficacy of LKT (Thomaz et al., 2018; Kafa et al., 2015) and of IPC (Feldman et al., 2012; Airaksinen et al., 1991) in the management of edemas.

Many modern research pointed out how lymphedema, of whatever nature it is, represents a much more complex disease than previously thought, indicating the necessity to integrate new strategies of control of the problem in terms of diagnostic and, mostly, therapeutic (Bjork and Hettrick, 2019; Van Cleave and Tilley, 2019).

Therefore, in a context in which the research of new approaches to lymphedema seems to be determinant to guarantee a progress in the management of the pathology, it appears to be necessary to evaluate alternative methods of treatment which, like LKT and IPC, can guarantee some therapeutic advantages in terms of efficacy and inexpensiveness of the treatment, at least as complements to the classic approach (Bjork and Hettrick, 2019; Van Cleave and Tilley, 2019).

Results of this study are encouraging, demonstrating the efficacy of LKT and IPC in the management of primary lymphedema within stage 2 of lower limbs, tools these characterized by some important advantages compared to few or null disadvantages and side effects.

It is clear how the simplicity of application, the null invasiveness and the inexpensiveness of LKT make this approach potentially viable option for the treatment of lower limbs lymphedema, although the right application from the operator appears to be a determinant factor to maximize alleged effects of the technique (Wu et al., 2015).

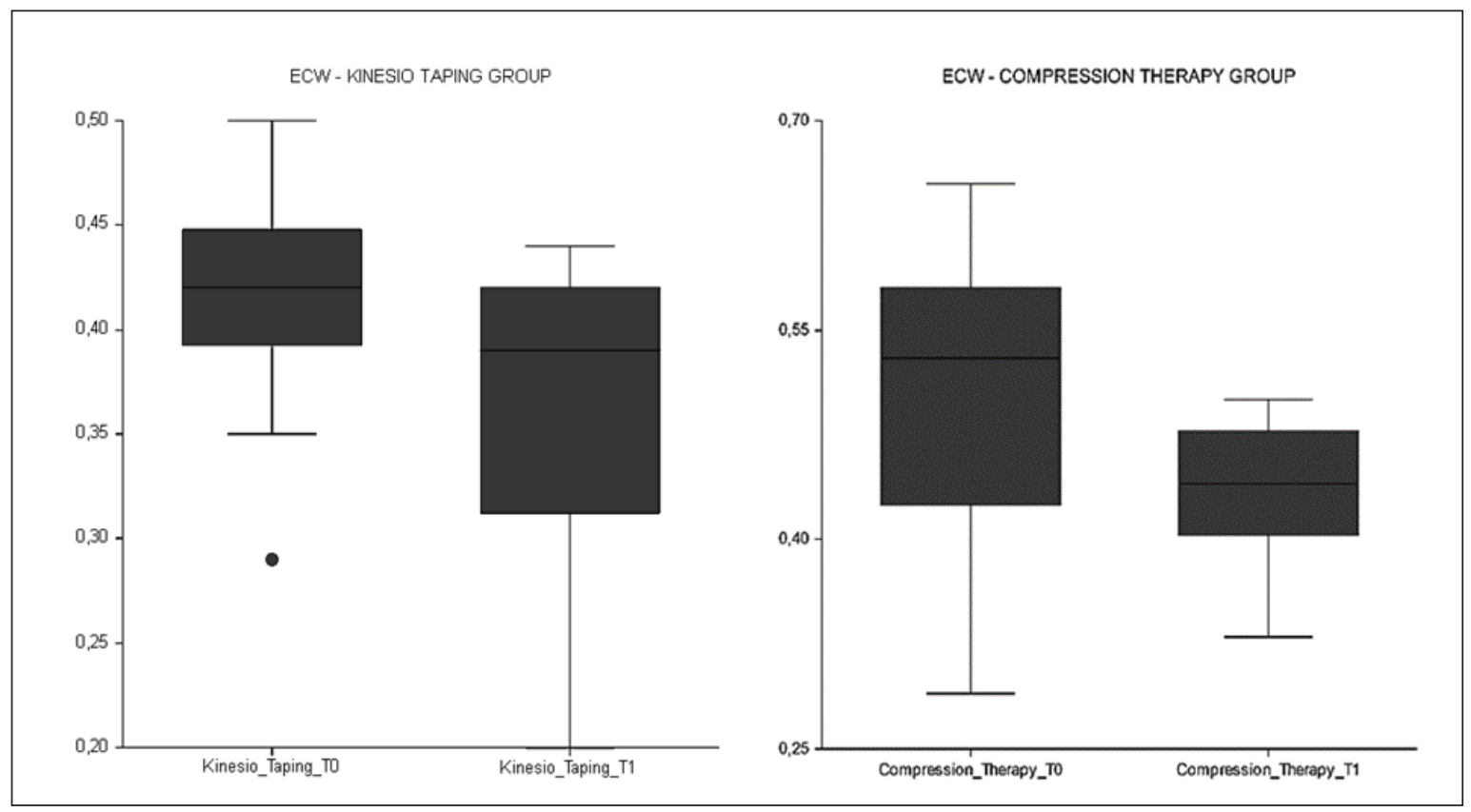

Fig. 4.1 , 4.2. - Statistical box plots of the mean variations of extracellular body water ECW in group A (LKT) and group B (IPC). 
Similarly, IPC is characterized by some advantages compared to other classical approaches for the management of lymphedema, resulting easier to wear and apply compared to compression elastic stockings and bandages (Tessari et al., 2018), as well as less challenging for the therapist compared to manual lymphatic drainage, inasmuch independent from the therapist dexterity and time available.

Moreover, a further advantage of modern IPC devices is the possibility to accurately personalize the treatment in terms of grade of compression. In general, the main disadvantages of IPC are represented by the possibly high cost of a device, especially as a homecare device, as well as the invasiveness in terms of time dedicated to the therapy in contrast to wearable systems like sock and bandages.

\section{CONCLUSIONS}

Is possible to affirm that both LKT and IPC are viable option for the management of primary lymphedema within stage 2 of lower limbs, both able to induce a volumetric reduction of the edema and the amelioration of the hydric balance of the body.

By virtue of the results obtained it could be interesting to explore the real efficacy of these methods setting a study with a larger sample size, possibly including a middle-term and long-term follow up and a direct comparison with a control group treated with classical compressive sock or bandage.

It would be also interesting, in our opinion, to evaluate the efficacy of a combination of the two studied treatments compared to the single methods, to eventually validate these methods as a viable complementary strategy for the management of lymphedema.

\section{REFERENCES}

1. Abu Khaled, M., McCutcheon, M. J., Reddy, S., Pearman, P. L., Hunter, G. R., \& Weinsier, R. L. (1988). Electrical impedance in assessing human body composition: the BIA method. The American journal of clinical nutrition, 47(5), 789-792.

2. Airaksinen, O., Partanen, K., Kolari, P. J., \& Soimakallio, S. (1991). Intermittent pneumatic compression therapy in posttraumatic lower limb edema: computed tomography and clinical measurements. Archives of physical medicine and rehabilitation, 72(9), 667670 .

3. Aldrich, M. B., Gross, D., Morrow, J. R., Fife, C. E., \& Rasmussen, J. C. (2017). Effect of pneumatic compression therapy on lymph movement in lymphedema-affected extremities, as assessed by near-infrared fluorescence lymphatic imaging. Journal of innovative optical health sciences, 10(02), 1650049.

4. Bjork, R., \& Hettrick, H. (2019). Lymphedema: New Concepts in Diagnosis and Treatment. Current Dermatology Reports, 8(4), 190-198.

5. Feldman, J. F., Stout, N. L., Wanchai, A., Stewart, B. R., Cormier, J. N., \& Armer, J. M. (2012). Intermittent pneumatic compression therapy: a systematic review. Lymphology, 45(1), 13-25.

6. Gurdal, S. O., Kostanoglu, A., Cavdar, I., Ozbas, A., Cabioglu, N., Ozcinar, B., et al. (2012). Comparison of intermittent pneumatic compression with manual lymphatic drainage for treatment of breast cancer-related lymphedema. Lymphatic research and biology, 10(3), 129-135

7. Kafa, N., Citaker, S., Omeroglu, S., Peker, T., Coskun, N., \& Diker, S. (2015). Effects of kinesiologic taping on epidermal-dermal distance, pain, edema and inflammation after experimentally induced soft tissue trauma. Physiotherapy theory and practice, $31(8)$, 556-561.

8. Kase, K., \& Stockheimer, K. R. (2006). Kinesio taping for lymphoedema and chronic swelling. Kinesio USA, LLC.

9. Kase, K. (1996). Development of Kinesio ${ }^{\wedge}<\mathrm{TM}>$ tape. Kinesio ${ }^{\wedge}<T M>$ Taping Perfect Manual.

10. Lee, B. B., Rockson, S. G., \& Bergan, J. (Eds.). (2018). Lymphedema: a concise compendium of theory and practice. Springer.

11. Pivetta, H. M. F., do Nascimento Petter, G., dos Santos, L. F., \& de Oliveira Martins, T. N. (2017). Efeitos do Kinesio Taping sobre o edema linfático. Fisioterapia Brasil, 18(3), 382-390.

12. Rockson, S. G. (2018). Intermittent pneumatic compression therapy. In Lymphedema (pp. 443-448). Springer, Cham.

13. te Slaa, A., Mulder, P., Dolmans, D., Castenmiller, P., Ho, G., \& Van der Laan, L. (2011). Reliability and reproducibility of a clinical application of a simple technique for repeated circumferential leg measurements. Phlebology, 26(1), 14-19.

14. Teixeira, L. N., Sandrin, F., Baggi, F., \& Simoncini, M. C. (2016). Linfotaping with Kinesio ${ }^{\circledR}$ Tape to manage and treat lymphedema patients: safety and tolerability are more important than efficacy? Supportive Care in Cancer, 24(8), 3279-3280.

15. Tessari, M., Tisato, V., Rimondi, E., Zamboni, P., \& Malagoni, A. M. (2018). Effects of intermittent pneumatic compression treatment on clinical outcomes and biochemical markers in patients at low mobility with lower limb edema. Journal of Vascular Surgery: Venous and Lymphatic Disorders, 6(4), 500-510.

16. Thomaz, J. P., Dias, T. D. S. M., \& Rezende, L. F. D. (2018). Efeito do uso do taping na redução do volume do linfedema secundário ao câncer de mama: revisão da literatura. Jornal Vascular Brasileiro, 17(2), 136-140.

17. Van Cleave, J. H., \& Tilley, C. P. (2019). Lymphedema Management. Oxford Textbook of Palliative Nursing, 14, 238.

18. Ward, L. C. (2006). Bioelectrical impedance analysis: proven utility in lymphedema risk assessment and therapeutic monitoring. Lymphatic research and biology, 4(1), 51-56.

19. Wu, W. T., Hong, C. Z., \& Chou, L. W. (2015). The kinesio taping method for myofascial pain control. Evidence-Based complementary and alternative medicine, 2015. 